\title{
ARTICLE OPEN \\ CaTe: a new topological node-line and Dirac semimetal
}

\author{
Yongping Du ${ }^{1}$, Feng Tang ${ }^{1}$, Di Wang ${ }^{1}$, Li Sheng ${ }^{1,2}$, Er-jun Kan ${ }^{3}$, Chun-Gang Duan ${ }^{4}$, Sergey Y. Savrasov ${ }^{5}$ and Xiangang Wan ${ }^{1,2}$
}

Combining first-principles calculations and effective model analysis, we predict that CaTe is a topological node-line semimetal in the absence of the spin-orbit coupling. Using a slab model, we obtain the nearly flat drumhead surface state near the Fermi level. When the spin-orbit coupling is included, three node lines will evolve into a pair of Dirac points along the $M-R$ line. These Dirac points are robust and protected by the $C_{4}$ rotation symmetry. Once this crystal symmetry is broken, the Dirac points will be eliminated, and the system becomes a strong topological insulator.

npj Quantum Materials (2017)2:3 ; doi:10.1038/s41535-016-0005-4

\section{INTRODUCTION}

Topological insulator ( $\mathrm{TI}$ ) has attracted broad interest in the past 10 years. $^{1-7}$ The unique property of $\mathrm{TI}$ is that it is an insulator in the bulk but has exotic metallic surface states due to the topological order. More recently, the research on topological states has been extended to three-dimensional (3D) semimetal systems. ${ }^{8-12}$ These systems could demonstrate many fascinating phenomena, such as Weyl fermion quantum transport and various Hall effects, ${ }^{13-16}$ consequently become the rising stars of the field. Up to now, three kinds of topological semimetals have been discovered, i.e., 3D Dirac semimetal (DSM), ${ }^{12,17-21}$ Weyl semimetal (WSM), ${ }^{8,9,22,23}$ and node-line semimetal (NLS). ${ }^{24-29}$ The 3D DSM has four-fold degeneracy point, which can be viewed as the kiss of two Weyl fermions with opposite chiralities in the Brillouin zone (BZ). Protected by the crystal symmetry and time reversal symmetry, 3D DSMs can be robust against external perturbations. Several materials have been theoretically predicted to be 3D DSMs ${ }^{12,17-21}$ and some of them have already been confirmed by experiments. $^{30-33}$ If one breaks the time reversal symmetry $8,22,23,34$ or the inversion symmetry, ${ }^{35-38}$ the 3D DSM will evolve into WSM. Very recently, the predictions about WSM state in TaAs family ${ }^{37,38}$ have been confirmed experimentally. ${ }^{39-42}$

Unlike DSM and WSM whose band crossing points distribute at separate $k$ points in the BZ, for a NLS the crossing points around the Fermi level form a closed loop. Several compounds have been proposed as NLSs, including Mackay-Terrones crystals, ${ }^{25}$ Bernal graphite ${ }^{43}$ hyperhoneycomb lattices, ${ }^{44}$ and antiperovskite $\mathrm{Cu}_{3} \mathrm{PdN}^{26,27}$ and $\mathrm{Cu}_{3} \mathrm{NZn}^{27}$ In the absence of spin-orbit coupling (SOC), time reversal symmetry together with inversion or mirror symmetry will guarantee the occurrence of node line in 3D BZ for systems with band inversion. ${ }^{25-27,37,45,46}$ Same with TI and WSM, NLS also has a characteristic surface state, namely, drumhead-like state. ${ }^{24-29}$ Such a 2D flat band surface state may become a route to achieve high temperature superconductivity. ${ }^{47,48}$ As a result, it is of great impetus to search new NLS.

In this study, based on first-principles calculations and effective model analysis, we propose that $\mathrm{CaTe}$ in $\mathrm{CsCl}$-type structure is a NLS with drumhead-like surface flat bands when the SOC is ignored. As shown in Fig. $1 \mathrm{~b}$, around the $M$ point, there are three node-line rings, which are perpendicular from one and another. When the SOC is included, these three node-line rings evolve into two Dirac points along the $M-R$ line. The Dirac points are robust and protected by the $C_{4}$ rotational symmetry. If this symmetry is broken, the system becomes a strong TI with $Z_{2}$ indices $(1 ; 000)$.

\section{RESULTS AND DISCUSSIONS}

As one member of the alkaline-earth chalcogenides, CaTe has attracted tremendous interests because of its technological applications ranging from catalysis to luminescence. ${ }^{49-53}$ CaTe undergoes a phase transition from $\mathrm{NaCl}$-type structure at ambient conditions to $\mathrm{CsCl}$-type structure at hydrostatic pressure about 33 GPa. ${ }^{49,50}$ The structure of CaTe in CsCl-type is shown in Fig. 1a. The space group of this phase is $P m \overline{3} m$ (NO. 221).

First, we calculate the band structure of $\mathrm{CaTe}$ and show the result without SOC in Fig. 2a. By checking the wave functions, we find that the valence bands and conduction bands are mainly contributed by the $5 p_{z}$ (blue) state of Te and $3 d_{z^{2}}$ (red) state of $C a$, respectively, as shown in Fig. 2a. The band inversion occurs at $M$ point, where the energy of Te- $5 p_{z}$ state is higher than the energy of $\mathrm{Ca}-3 d_{z^{2}}$ state by about $0.75 \mathrm{eV}$. Interestingly, this kind of band inversion is not caused by the SOC, which is different from most of topological materials. ${ }^{1,2}$ We calculate the electronic structure of CaTe by applying tensile strain to check the origin of band inversion at $M$ point. The energy difference between the Te- $5 p_{z}$ state and Ca- $3 d_{z^{2}}$ state decreases as the tensile strain increases. When the strain is larger than $5 \%$, the band inversion at $M$ point disappears. Therefore, the band inversion originates from the crystal field effect. With the time reversal and space inversion symmetry, such band inversion will result in node lines as proved in Weng et al. ${ }^{25}$

The effective Hamiltonian for the node line around $M$ point can be established by using the $\vec{k} \cdot \vec{p}$ method. Considering the crystal symmetry at $M$ point and time reversal symmetry, the effective

\footnotetext{
${ }^{1}$ National Laboratory of Solid State Microstructures and Department of Physics, Nanjing University, Nanjing 210093, China; ${ }^{2}$ Collaborative Innovation Center of Advanced Microstructures, Nanjing University, Nanjing 210093, China; ${ }^{3}$ Key Laboratory of Soft Chemistry and Functional Materials (Ministry of Education), and Department of Applied Physics, Nanjing University of Science and Technology, Nanjing, Jiangsu 210094, P. R. China; ${ }^{4}$ Key Laboratory of Polar Materials and Devices, Ministry of Education, East China Normal University, Shanghai 200062, China and ${ }^{5}$ Department of Physics, University of California, Davis, One Shields Avenue, Davis, CA 95616, USA Correspondence: Xiangang Wan (xgwan@nju.edu.cn)
}

Received: 12 July 2016 Revised: 30 October 2016 Accepted: 22 November 2016 Published online: 23 January 2017 


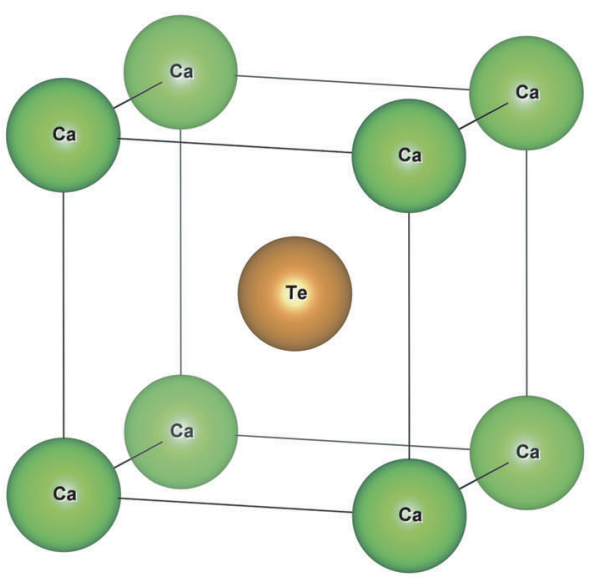

(a)

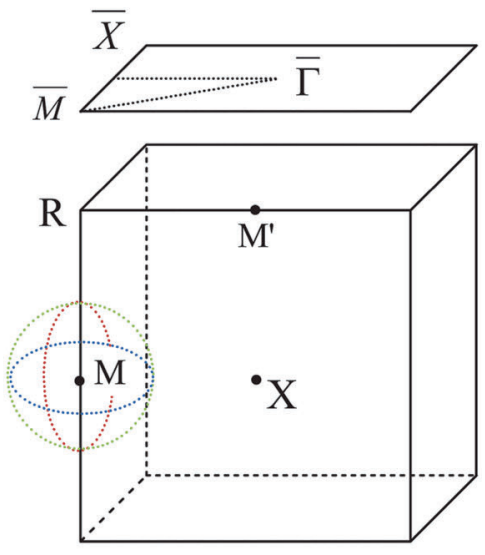

(b)

Fig. 1 a Crystal structure of $\mathrm{CaTe}$ in $\mathrm{CsCl}$ type phase. b The 3D BZ and projected (001) two dimensional (2D) BZ of CaTe. Three dash circles are the scheme of the three line nodes around the $M$ point. The blue circle is parallel to $k_{z}=0$ plane, red circle is parallel to $k_{x}=0$ plane, and the green circle is parallel to $k_{y}=0$ plane

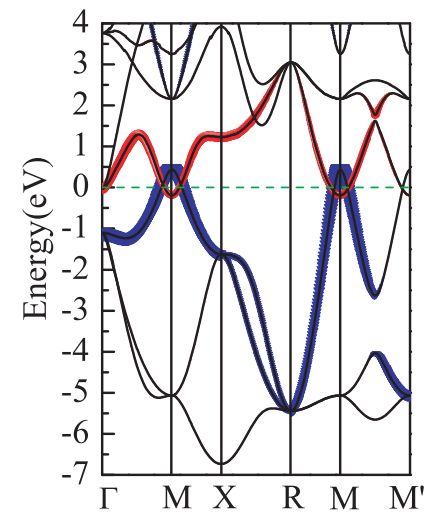

(a)

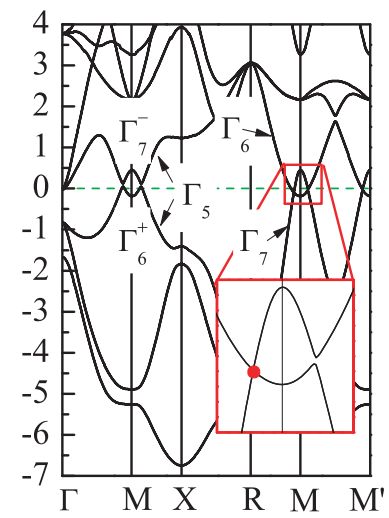

(b)

Fig. 2 a Electronic structure of CaTe without SOC. The weights of Te- $5 p_{z}\left(\mathrm{Ca}-3 d_{z^{2}}\right)$ state is proportional to the width of blue (red) curves. b Electronic structure of CaTe with SOC. Along the $\Gamma-M, M-X$, and $M-M^{\prime}$, a small gap is opened. The Dirac point at the $M-R$ line is protected by the $C_{4}$ rotational symmetry. The inset is the detailed structure around the $M$ point (see main text for detailed description)

Hamiltonian can be written as the following form:

$H(\vec{k})=g_{0}(\vec{k}) \sigma_{0}+g_{x}(\vec{k}) \sigma_{x}+g_{z}(\vec{k}) \sigma_{z}$,

where $\sigma_{x}$ and $\sigma_{z}$ are the Pauli matrices, $\sigma_{0}$ is the unit matrix. $g_{0}(\vec{k})=M_{0}-B_{0}\left(k_{x}^{2}+k_{y}^{2}\right)-C_{0} k_{z}^{2} g_{x}(\vec{k})=A k_{x} k_{y} k_{z}, g_{z}(\vec{k})=M_{z}-$ $B_{z}\left(k_{x}^{2}+k_{y}^{2}\right)-C_{z} k_{z}^{2}$. This system has both the time reversal symmetry and the inversion symmetry, thus the component $\sigma_{y}$ is zero. ${ }^{25}$ The eigenvalues of this $2 \times 2$ effective Hamiltonian are $E(\vec{k})=g_{0}(\vec{k}) \pm \sqrt{g_{x}^{2}(\vec{k})+g_{z}^{2}(\vec{k})}$. When $g_{x}(\vec{k})=0$ and $g_{z}(\vec{k})=0$, the nodal line will emerge. It can be easily checked that the equation $g_{z}(k)=0$ has solution only when $M_{z} B_{z}>0$ and $M_{z} C_{z}>0$. Note that such conditions apply also for the band inversion. On the other hand, $g_{x}(\vec{k})=0$ confines the node lines in three mutually perpendicular planes (namely, $k_{x}=0, k_{y}=0$, and $k_{z}=0$ planes ) as illustrated in Fig. $1 \mathrm{~b}$. Due to the fact that $g_{0}(\vec{k})$ does

not equal to 0 , which breaks the electron-hole symmetry, the node lines have finite energy dispersion.

When the SOC is considered, however, three node lines evolve into two Dirac points at the $M-R$ line as shown in Fig. $2 \mathrm{~b}$. At $M$ point, the two states near the Fermi level belong to irreducible representation $\Gamma_{7}^{-}$and $\Gamma_{6}^{+}$, respectively. While along the $M-X$ line, two bands have the same irreducible representation $\left(\Gamma_{5}\right)$ as shown in Fig. $2 b$, they can hybridize with each other, opening a small gap (about $50 \mathrm{meV}$ ). For both the $\Gamma-M$ line and the $M-M^{\prime}$ line, the two bands around the Fermi level also belong to the same irreducible representation, thus there is no band crossing along the $\Gamma-M$ line and the $M-M^{\prime}$ line. We point out here that since the band splitting is determined by the SOC, one therefore can achieve the NLS by doping the light atoms such as $\mathrm{Se}, \mathrm{S}$.

Along the $M-R$ line, which reserves the $C_{4}$ rotation symmetry, the two states with $\Gamma_{7}^{-}$and $\Gamma_{6}^{+}$at $M$ point evolve into $\Gamma_{7}$ and $\Gamma_{6}$. Consequently, the hybridization between these two bands is forbidden, generating a Dirac point as shown in Fig. 2b. When the $C_{4}$ rotational symmetry is broken, e.g., by strain effect, the band crossing point will disappear, and this 3D DSM will become a strong $\mathrm{TI}$ with topological indices $Z_{2}$ to be $(1 ; 000)$.

To better understand the band inversion at $M$ point and the topological property of this system, we derive a low-energy effective Hamiltonian at $M$ point based on the projection-operator method. ${ }^{20}$ The $M$ point has the $D_{4 h}$ symmetry and also the time reversal symmetry. As discussed above, at $M$ point, $\Gamma_{7}^{-}$symmetry state has angular momentum $j_{z}= \pm 3 / 2$ and $\Gamma_{6}^{+}$symmetry state has angular momentum $j_{z}= \pm 1 / 2$. Therefore using the basis of $\left(\left|j_{z}=-\frac{1}{2}\right\rangle_{d},\left|j_{z}=+\frac{1}{2}\right\rangle_{d},\left|j_{z}=-\frac{3}{2}\right\rangle_{p},\left|j_{z}=+\frac{3}{2}\right\rangle_{p}\right)$, the effective Hamiltonian around $M$ point can be written as (see Supplementary information (S1) for details):

$$
H_{\text {eff }}=
$$$$
\left(\begin{array}{cccc}
M_{1}(\vec{k}) & 0 & A k_{+}+B(\vec{k}) & D(\vec{k}) \\
0 & M_{1}(\vec{k}) & D^{*}(\vec{k}) & -A k_{-}-B^{*}(\vec{k}) \\
A k_{-}+B^{*}(\vec{k}) & D(\vec{k}) & M_{2}(\vec{k}) & 0 \\
D^{*}(\vec{k}) & -A k_{+}-B(\vec{k}) & 0 & M_{2}(\vec{k}),
\end{array}\right)
$$

where $\quad M_{1}(\vec{k})=M_{10}+M_{11}\left(k_{x}^{2}+k_{y}^{2}\right)+M_{12} k_{z}^{2} \quad M_{2}(\vec{k})=M_{20}+$ $M_{21}\left(k_{x}^{2}+k_{y}^{2}\right)+M_{22} k_{z}^{2} \quad B(\vec{k})=B_{1} k_{+} k_{z}^{2}+B_{2}\left(k_{x}^{3}+i k_{y}^{3}\right)+i B_{3} k_{-} k_{x} k_{y}$, $D(k)=D_{1}\left(k_{x}^{2}-k_{y}^{2}\right) k_{z}+i D_{2} k_{x} k_{y} k_{z}, k_{ \pm}=k_{x} \pm i k_{y}$. Along the $k_{z}$ axis 

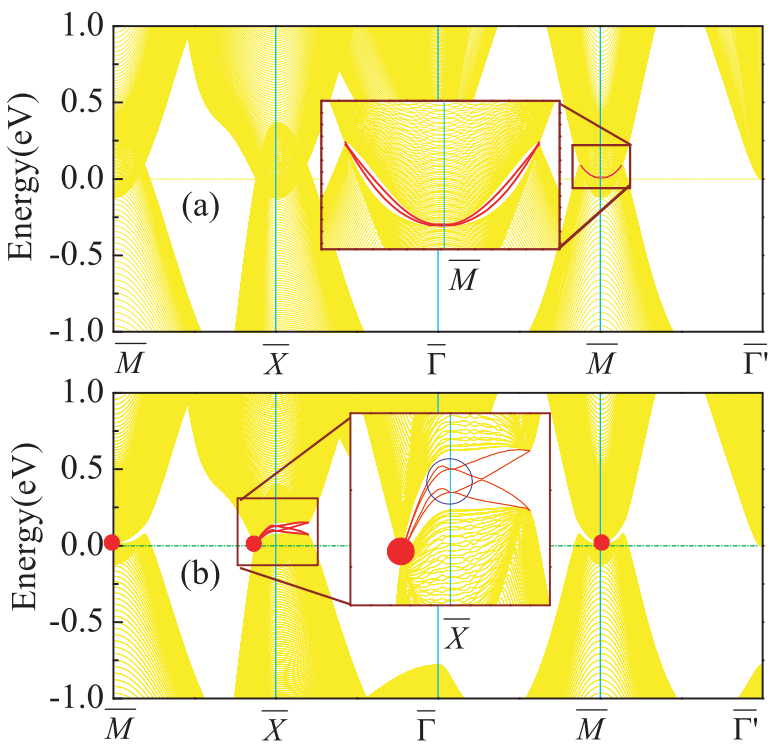

Fig. 3 The surface states of CaTe (001): a without and $\mathbf{b}$ with the SOC. In a, the flat surface state around the Fermi level is denoted by red color. The inset is the detailed band structure around the $\bar{M}$ point. In $\mathbf{b}$, the red dots are the projected bulk Dirac nodes. The red lines between the bulk gap at $\bar{X}$ point are the surface states and the blue circle denotes the surface Dirac cones. The inset is the detailed band structure around the $\bar{X}$ point

(where $k_{x}=0, k_{y}=0$ ), the effective Hamiltonian is diagonal, and the eigenvalues are $E(\vec{k})=M_{1}(\vec{k})$ and $E(\vec{k})=M_{2}(\vec{k})$. As mentioned above, the Dirac point is on the $M-R$ line, thus it is interesting to discuss the effective model along this line. Since there is the band inversion between $\left|j_{z}= \pm \frac{1}{2}\right\rangle_{d}$ and $\left|j_{z}= \pm \frac{3}{2}\right\rangle_{p}$ at $M$ point, it is easy to obtain that $M_{10}<M_{20}, M_{22}<0<M_{12}$, and the Dirac points locate at $\vec{k}_{c}=\left(\frac{\pi}{a}, \frac{\pi}{a}, k_{z c}= \pm \sqrt{\frac{M_{20}-M_{10}}{M_{12}-M_{22}}}\right)$. Neglecting the high-order terms, $E\left(\vec{k}_{c}+\overrightarrow{\delta k}\right)$ can be expressed as $\left(M_{12}+M_{22}\right) k_{z c} \delta k_{z} \pm \sqrt{\left(M_{12}-M_{22}\right)^{2} k_{z c}^{2} \delta k_{z}^{2}+A^{2}\left(\delta k_{x}^{2}+\delta k_{y}^{2}\right)}$, where $\delta k_{x, y, z}$ are the small displacements from $\vec{k}_{c}$. In the vicinity of $\vec{k}_{c}$, the band dispersion is linear, thus our effective Hamiltonian describes exactly the 3D massless Dirac fermions.

The band inversion at $M$ point and the Dirac nodes in CaTe suggest the existence of topological nontrivial surface state. Figure $3 a$, b shows the surface state of CaTe (001) surface without/ with the SOC, respectively. When the SOC is absent, the system is a NLS, and possesses nearly flat surface band around the Fermi energy. As shown in Fig. 3a, our numerical results find that the nearly flat surface "drumhead" state appears in the interiors of the projected nodal line rings on the (001) surface around the $\bar{M}$ point. Since the slab we used has two surfaces, there are two surface states as shown in the red lines in the Fig. 3a. The particle-hole symmetry is broken by the non-zero term $g_{0}(\vec{k})$, thus these two surface bands are not perfectly flat with about $70 \mathrm{meV}$ bandwidth. This type of 2D flat bands is proposed as a novel route to achieve high temperature superconductivity. ${ }^{47,48}$

When the SOC is included, three node lines are gapped out and evolve into a pair of Dirac points along the $M-R$ line, thus the NLS becomes a 3D DSM. There is a bulk Dirac node projected on the $\bar{M}$ point (the red dot) as shown in Fig. 3b. Along the $\bar{M}-\bar{X}$, there is also a projected bulk Dirac node, which locates near the $\bar{X}$ point denoted by red dots. Figure $3 \mathrm{~b}$ clearly shows the gapped bulk states along the $\bar{\Gamma}-\bar{X}$ direction and the existence of surface Dirac cones (in the blue circle) due to the topologically nontrivial $Z_{2}$ indices, same as the case of $\mathrm{Na}_{3} \mathrm{Bi}^{18}$ and $\mathrm{Cd}_{3} \mathrm{As}_{2}$. ${ }^{19}$
In summary, based on the first-principles calculations and effective model analysis, we suggest that $\mathrm{CaTe}$ in $\mathrm{CsCl}$-type structure is a NLS when the SOC is ignored. There are three nodeline rings that are perpendicular from one and another around $M$ point. With band inversion at $M$ point, this NLS is protected by the time reversal symmetry and space inversion symmetry. When the SOC is included, three node-line rings become a pair of Dirac points. These Dirac nodes are robust and protected by the $C_{4}$ crystal symmetry and the system becomes a DSM. Experimentally, to achieve the NLS state, one may consider to decrease the SOC effect by doping the light atoms such as Se, S into the system.The simple cubic structure of CaTe makes this compound much easier synthesized. On the other hand, the clean Fermi surface of CaTe results in a simple surface state, which brings benefit to the measurement of flat-band surface state. Therefore, CaTe is a promising candidate material of NLS.

\section{METHODS}

The electronic band structure calculations have been carried out using the full potential linearized augmented plane-wave method as implemented in the WIEN2K package. ${ }^{54}$ To obtain accurate band inversion strength and band order, the modified Becke-Johnson exchange potential together with the local-density approximation for the correlation potential (MBJLDA) has been applied. ${ }^{55}$ We also check the electronic structure with other exchange potentials like the local-density approximation Perdew-BurkeErnzerhof parametrization of the generalized gradient approximation. ${ }^{56}$ The results show no significant differences. The plane-wave cutoff parameter $R_{\mathrm{MT}} K_{\max }$ is set to be 7 and a $16 \times 16 \times 16$ mesh is used for the BZ integral. The SOC is treated using the second-order variational procedure.

To study the surface states in CaTe, we use a 200-unit-cells-thick (001) slab with the top (bottom) surface terminated by $\mathrm{Ca}(\mathrm{Te})$ atoms. The surface state is then calculated using the tight-binding method. The hopping parameters are determined from the maximally localized Wannier functions, ${ }^{57}$ which are projected from the Bloch state derived from the first-principles calculations.

\section{ACKNOWLEDGEMENTS}

The work was supported by the National Key Project for Basic Research of China (Grant No. 2014CB921104), NSFC under Grants No. 11525417, 11374147, 51572085. The project is also funded by Priority Academic Program Development of Jiangsu Higher Education Institutions. S.S. was supported by NSF DMR (Grant No. 1411336). Y. D. is supported by the program B for Outstanding Ph.D. candidate of Nanjing University.

\section{AUTHOR CONTRIBUTIONS}

X.W. notices the line node and Dirac points in this system. Y.D. performs the firstprinciples calculations. Y.D., F.T., and D.W. do the symmetry analysis and the model. X. $W$. interprets the numerical results and writes the paper. All authors contribute to editing the manuscript.

\section{COMPETING INTERESTS}

The authors declare no competing financial interests.

\section{REFERENCES}

1 Hasan, M. Z. \& Kane, C. L. Colloquium: topological insulators. Rev. Mod. Phys. 82, 3045-3067 (2010)

2 Qi, X. L. \& Zhang, S. C. Topological insulators and superconductors. Rev. Mod. Phys. 83, 1057-1110 (2011).

3 Kane, C. L. \& Mele, E. J. Quantum spin hall effect in graphene. Phys. Rev. Lett. 95, 226801 (2005).

4 Kane, C. L. \& Mele, E. J. $Z_{2}$ topological order and the quantum spin hall effect. Phys. Rev. Lett. 95, 146802 (2005).

5 Bernevig, B. A. \& Zhang, S. C. Quantum spin hall effect. Phys. Rev. Lett. 96, 106802 (2006). 
6 Bernevig, B. A., Hughes, T. L. \& Zhang, S. C. Quantum spin hall effect and topological phase transition in HgTe quantum wells. Science 314, 1757-1761 (2006).

7 Zhang, H. et al. Topological insulators in $\mathrm{Bi}_{2} \mathrm{Se}_{3}, \mathrm{Bi}_{2} \mathrm{Te}_{3}$ and $\mathrm{Sb}_{2} \mathrm{Te}_{3}$ with a single Dirac cone on the surface. Nat. Phys. 5, 438-442 (2009).

8 Wan, X. G., Turner, A. M., Vishwanath, A. \& Savrasov, S. Y. Topological semimetal and Fermi-arc surface states in the electronic structure of pyrochlore iridates. Phys. Rev. B 83, 205101 (2011).

9 Balents, L. Viewpoint: Weyl electrons kiss. Physics 4, 36 (2011).

10 Ando, Y. Topological insulator materials. J. Phys. Soc. Jpn. 82, 102001 (2013).

11 Yang, K. Y., Lu, Y. M. \& Ran, Y. Quantum hall effects in a Weyl semimetal: possible application in pyrochlore iridates. Phys. Rev. B 84, 075129 (2011).

12 Wehling, T. O., Black-Schaffer, A. M. \& Balatsky, A. V. Dirac materials. Adv. Phys. 63, 1-76 (2014).

13 Hosur, P. \& Qi, X. L. Recent developments in transport phenomena in Weyl semimetals. Compt. Rend. Phys. 14, 857-870 (2013).

14 Zyuzin, A. A. \& Burkov, A. A. Topological response in Weyl semimetals and the chiral anomaly. Phys. Rev. B 86, 115133 (2012).

15 Vazifeh, M. M. \& Franz, M. Electromagnetic response of Weyl semimetals. Phys. Rev. Lett. 111, 027201 (2013).

16 Parameswaran, S. A., Grover, T., Abanin, D. A., Pesin, D. A. \& Vishwanath, A. Probing the chiral anomaly with nonlocal transport in three-dimensional topological semimetals. Phys. Rev. X 4, 031035 (2014).

17 Young, S. M. et al. Dirac semimetal in three dimensions. Phys. Rev. Lett. 108, 140405 (2012).

18 Wang, Z. et al. Dirac semimetal and topological phase transitions in $A_{3} B i(A=N a, K$, Rb). Phys. Rev. B 85, 195320 (2012).

19 Wang, Z., Weng, H. M., Wu, Q., Dai, X. \& Fang, Z. Three-dimensional Dirac semimetal and quantum transport in $C_{3} A s_{2}$. Phys. Rev. B 88, 125427 (2013).

$20 \mathrm{Du}, \mathrm{Y}$. et al. Dirac and Weyl semimetal in $\mathrm{XYBi}(\mathrm{X}=\mathrm{Ba}, \mathrm{Eu} ; \mathrm{Y}=\mathrm{Cu}, \mathrm{Ag}$ and $\mathrm{Au})$. Sci. Rep. 5, 14423 (2015).

21 Gibson, Q. D. et al. Three-dimensional Dirac semimetals: design principles and predictions of new materials. Phys. Rev. B 91, 205128 (2015).

$22 \mathrm{Xu}$, G., Weng, H. M., Wang, Z., Dai, X. \& Fang, Z. Chern semimetal and the quantized anomalous hall effect in $\mathrm{HgCr}_{2} \mathrm{Se}_{4}$. Phys. Rev. Lett. 107, 186806 (2011).

23 Burkov, A. A. \& Balents, L. Weyl semimetal in a topological insulator multilayer. Phys. Rev. Lett. 107, 127205 (2011).

24 Burkov, A. A., Hook, M. D. \& Balents, L. Topological nodal semimetals. Phys. Rev. B 84, 235126 (2011).

25 Weng, H. et al. Topological node-line semimetal in three-dimensional graphene networks. Phys. Rev. B 92, 045108 (2015).

26 Yu, R., Weng, H., Fang, Z., Dai, X. \& Hu, X. Topological node-line semimetal and Dirac semimetal state in antiperovskite $\mathrm{Cu}_{3} P d N$. Phys. Rev. Lett. 115, 036807 (2015).

27 Kim, Y., Wieder, B. J., Kane, C. L. \& Rappe, A. M. Dirac line nodes in inversionsymmetric crystals. Phys. Rev. Lett. 115, 036806 (2015).

28 Yang, S. Y., Pan, H. \& Zhang, F. Dirac and Weyl superconductors in three dimensions. Phys. Rev. Lett. 113, 046401 (2014).

29 Chen, Y. P. et al. Nanostructured carbon allotropes with Weyl-like loops and points. Nano Lett. 15, 6974-6978 (2015).

30 Jeon, S. et al. Landau quantization and quasiparticle interference in the threedimensional Dirac semimetal $\mathrm{Cd}_{3} A s_{2}$. Nat. Mater. 13, 851-856 (2014).

31 Neupane, M. et al. Observation of a three-dimensional topological Dirac semimetal phase in high-mobility $\mathrm{Cd}_{3} A s_{2}$. Nat. Commun. 5, 3786 (2014).

$32 \mathrm{Liu}, \mathrm{Z}$. K. et al. Discovery of a three-dimensional topological Dirac semimetal, $\mathrm{Na}_{3} \mathrm{Bi}$. Science 343, 864-867 (2014).

$33 \mathrm{Liu}, \mathrm{Z}$. K. et al. A stable three-dimensional topological Dirac semimetal $\mathrm{Cd}_{3} \mathrm{As}_{2}$. Nat. Mater. 13, 677-681 (2014).

34 Wan, X., Vishwanath, A. \& Savrasov, S. Y. Computational design of Axion insulators based on 5d spinel compounds. Phys. Rev. Lett. 108, 146601 (2012).

35 Halasz, G. B. \& Balents, L. Time-reversal invariant realization of the Weyl semimetal phase. Phys. Rev. B 85, 035103 (2012).
36 Liu, J. \& Vanderbilt, D. Weyl semimetals from noncentrosymmetric topological insulators. Phys. Rev. B 90, 155316 (2014).

37 Weng, H. M., Fang, C., Fang, Z., Bernevig, B. A. \& Dai, X. Weyl semimetal phase in noncentrosymmetric transition-metal monophosphides. Phys. Rev. X 5, 011029 (2015).

38 Huang, S. M. et al. A Weyl Fermion semimetal with surface Fermi arcs in the transition metal monopnictide TaAs class. Nat. Commun. 6, 7373 (2015).

39 Lv, B. Q. et al. Experimental discovery of Weyl semimetal TaAs. Phys. Rev. X 5, 031013 (2015).

40 Lv, B. Q. et al. Observation of Weyl nodes in TaAs. Nat. Phys. 11, 724-727 (2015).

$41 \mathrm{Xu}, \mathrm{S}$. Y. et al. Discovery of a Weyl fermion semimetal and topological Fermi arcs. Science 349, 613-617 (2015).

$42 \mathrm{Xu}, \mathrm{S}$. Y. et al. Discovery of a Weyl fermion state with Fermi arcs in niobium arsenide. Nat. Phys. 11, 748-754 (2015).

43 Mikitik, G. P. \& Sharlai, Y. V. Band-contact lines in the electron energy spectrum of graphite. Phys. Rev. B 73, 235112 (2006).

44 Mullen, K., Uchoa, B. \& Glatzhofer, D. T. Line of Dirac nodes in hyperhoneycomb lattices. Phys. Rev. Lett. 115, 026403 (2015).

45 Xie, L. S. et al. A new form of $\mathrm{Ca}_{3} \mathrm{P}_{2}$ with a ring of Dirac nodes. . APL Mater. 3, 083602 (2015).

46 Zeng, M., et al. Topological semimetals and topological insulators in rare earth monopnictides. Preprint at https://arxiv.org/abs/1504.03492 (2015).

47 Heikkila, T. T. \& Volovik, G. E. Flat bands as a route to high-temperature superconductivity in graphite. Preprint at https://arxiv.org/abs/1504.05824 (2015).

48 Kopnin, N. B., Heikkila, T. T. \& Volovik, G. E. High-temperature surface superconductivity in topological flat-band systems. Phys. Rev. B 83, 220503 (2011).

49 Zimmer, H. G., Winzen, H. \& Syassen, K. High-pressure phase transitions in CaTe and SrTe. Phys. Rev. B 32, 4066-4070 (1985).

50 Luo, H., Greene, R. G., Ghandehari, K., Li, T. \& Ruoff, A. L. Structural phase transformations and the equations of state of calcium chalcogenides at high pressure. Phys. Rev. B 50, 16232-16237 (1994).

51 Dadsetani, M. \& Pourghazi, A. Optical properties of strontium monochalcogenides from first principles. Phys. Rev. B 73, 195102 (2006).

52 Boucenna, S. et al. High pressure induced structural, elastic and electronic properties of Calcium Chalcogenides $\mathrm{CaX}(\mathrm{X}=\mathrm{S}$, Se and $\mathrm{Te}$ ) via first-principles calculations. Comput. Mater. Sci. 68, 325-334 (2013).

53 Varshneya, D., Rathore, V., Kinge, R. \& Singh, R. K. High-pressure induced structural phase transition in alkaline earth $\mathrm{CaX}(\mathrm{X}=\mathrm{S}$, Se and Te) semiconductors: $\mathrm{NaCl}-$ type (B1) to CsCl-type (B2). J. Alloys Compd. 484, 239-245 (2009).

54 Blaha, P., Schwarz, K., Madsen, G. K. H., Kvasnicka, D., \& Luitz, J. in WIEN2K: An Augmented Plane Wave + Local Orbitals Program for Calculating Crystal Properties (ed Schwarz, K.) (Vienna University of Technology, 2001).

55 Tran, F. \& Blaha, P. Accurate band gaps of semiconductors and insulators with a semilocal exchange-correlation potential. Phys. Rev. Lett. 102, 226401 (2009).

56 Perdew, J. P., Burke, K. \& Ernzerhof, M. Generalized gradient approximation made simple. Phys. Rev. Lett. 77, 3865-3868 (1996).

57 Marzari, N., Mostofi, A. A., Yates, J. R., Souza, I. \& Vanderbilt, D. Maximally localized Wannier functions: theory and applications. Rev. Mod. Phys. 84, 1419-1475 (2012)

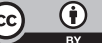

This work is licensed under a Creative Commons Attribution 4.0 International License. The images or other third party material in this article are included in the article's Creative Commons license, unless indicated otherwise in the credit line; if the material is not included under the Creative Commons license, users will need to obtain permission from the license holder to reproduce the material. To view a copy of this license, visit http://creativecommons.org/licenses/by/ $4.0 /$

(c) The Author(s) 2017

Supplementary Information accompanies the paper on the npj Quantum Materials website (doi:10.1038/s41535-016-0005-4). 